\title{
Preparation of Ceramic Humidity Sensors by Electrospinning and Sintering: a Promising Alternative
}

\author{
Evando S Araújo*, Victor N S Leão, Sandy R Monteiro, Ramiro P Guimarães, Allef F R Barbosa and Mayane M \\ Nascimento
}

Research Group on Electro spinning and Nanotechnology Applications-GPEANano, Brazil

*Corresponding author: Evando S Araújo, Research Group on Electrospinning and Nanotechnology Applications (GPEA-Nano), 48902-300, JuazeiroBA, Brazil

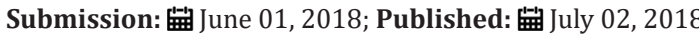

Abstract

Humidity is a critical environmental parameter for several production processes and its sensing is of great importance for maintaining the quality of goods and products. This work shows an alternative method for the production of ceramic nanocomposites (using the electro spinning and sintering techniques) for application as humidity sensors.

Keywords: Humidity sensors; Electro spinning; Preparation; Ceramic nanocomposites

\section{Introduction}

Humidity is a parameter of great importance for a better quality in the production, storage and transportation of food, goods and medicinal products by industry, among others. In this sense, relative humidity (RH) is the most frequently used parameter for assessing moisture [1]. Humidity sensors are usually made of the polymer or ceramics. Ceramics present superior performance because they have high chemical/structural stability and fast electrical response as a function of $\mathrm{RH}$, operating over a wide range of temperatures. The changes in the electrical response of these sensors are related to the chemisorbed and physisorbed layers of water molecules on the surface of the ceramic particles and to the capillary condensation of water in the microscopic pores between the particles (with the progressive increase of $\mathrm{RH}$ ) [1]. The selection of oxides, the grain sizes, distribution and shape of the pores, the presence of selective do pants and the production method are decisive factors for producing a device with high sensitivity to humidity [1-3].

In this context, heterogeneous ceramic nanostructures of metallic oxide are materials of great technological interest for the production of more efficient sensors due to excellent electronic and optical properties, high micro structural porosity, regular pore and grain distributions and more reactive surface with water (greater surface area of action/volume) [2,3].

One of the most commonly used metal oxides in humidity sensors is anatase titanium dioxide $\left(\mathrm{TiO}_{2}\right)(\sim 3.2 \mathrm{eV}$ band gap). Other metal oxides such as zinc oxide ( $\mathrm{ZnO})$, copper oxide ( $\mathrm{CuO})$, tungsten trioxide $\left(\mathrm{WO}_{3}\right)$, vanadium pentoxide $\left(\mathrm{V}_{2} \mathrm{O}_{5}\right)$, niobium pentoxide $\left(\mathrm{Nb}_{2} \mathrm{O}_{5}\right)$ and indium oxide $\left(\operatorname{In}_{2} \mathrm{O}_{3}\right)$ have been used as $\mathrm{TiO}_{2}$ do pants. The $\mathrm{TiO}_{2}: \mathrm{WO}_{3}$ composition (1:1 in mol) guarantees a higher conductivity over pure $\mathrm{TiO}_{2}$, while do pants such as $\mathrm{V}_{2} \mathrm{O}_{5}$ and $\mathrm{Nb}_{2} \mathrm{O}_{5}$ retard the thermally activated phase transition from anatase to retile $\mathrm{TiO}_{2}$, which favours sensitivity to $\mathrm{RH}[2,3]$. In the last years, these materials have been evaluated as humidity sensors, from a mixture between $\mathrm{TiO}_{2}$ and $\mathrm{WO}_{3}$ doped with $\mathrm{V}_{2} \mathrm{O}_{5}, \mathrm{Nb}_{2} \mathrm{O}_{5}, \mathrm{ZnO}$ or $\mathrm{CuO}$, in the form of volumetric pellets (length $\mathrm{x}$ width $\mathrm{x}$ height) $[2,3]$.

\section{Humidity sensors prepared by electro spinning and sin- tering}

An alternative to increase the efficiency of these moisture sensors and decrease the costs to produce them is to further increase the surface area/volume ratio by dispersing the oxides in polymer nanofibers produced by the electro spinning technique and subsequent sintering of the resulting material $[4,5]$. In the context of this work, initially, the metal oxides are encapsulated in polymer nanofibers through the electro spinning technique (applying a potential difference of $20 \mathrm{kV}$ ); and then, the resulting material is arranged on an inter digitated electrode and sintered $\left(500{ }^{\circ} \mathrm{C}\right)$ to eliminate the organic phase and obtain the ceramic sensor (Figure 1). Nanofibers act as a polymeric template to ensure adequate distribution of the oxides on the electrode, while the sintering process is used to form heterogeneous ceramic nanostructures with lower band gap, which favours the conduction processes in the material under the action of RH $[4,5]$. 


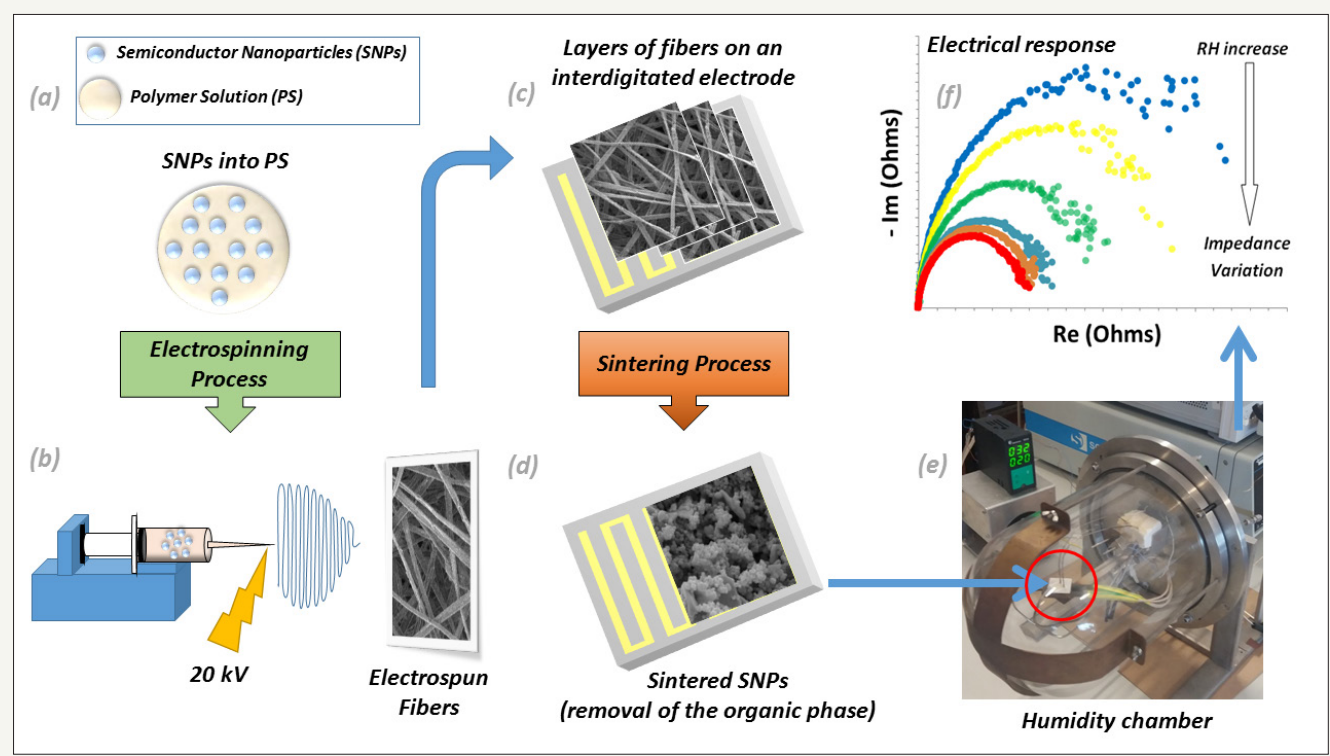

Figure 1: Step by step preparation of humidity sensors by electro spinning and sintering process: (a) oxide nanoparticles into polymer solution for the electro spinning process; (b) electro spun fibers collected as action of a $20 \mathrm{kV}$ voltage; (c) layers of fibers disposed on a inter digitated electrode; (d) sintered nanoparticles on a inter digitated electrode (removal of polymeric phase) used as humidity sensor; (e) humidity sensor in a humidity chamber for electrical characterization; and (f) electrical response of the ceramic nanocomposites as function of $\mathrm{RH}$.

In summary, with this new preparation route it is possible to produce thin films of ceramic nanoparticles, using smaller amounts of material than that used to produce disks or pellets, with a larger surface area, without loss of sensorial quality and cheaper. In addition, the electro spinning technique is also promising for moisture sensor production because it offers more advantages such as flexibility in the choice of materials and design, low cost in automation, mass production and good reproducibility of results. The studies also show that the fabrication technique, the sintering temperature and the do pant concentration are decisive factors for increasing the efficiency of the sensors [5].

\section{Conclusion and Perspectives}

This work shows to the scientific community the possibility of producing heterogeneous ceramic nanostructures for application as moisture sensors through the electro spinning and sintering techniques. The new preparation route can generate sensors that are more sensitive to variations in moisture when compared to the same materials produced by other methods. The best obtained results characterize the presented proposal as an open field of research for the synthesis and micro structural characterization of these electronic materials for the industry.

\section{Acknowledgement}

The authors would like thanks to Dr. Helinando Oliveira and Dr Pedro Faia for their support and teachings..

\section{References}

1. Traversa E (1995) Ceramic sensors for humidity detection: the stateof-the-art and future developments. Sensors and Actuators B: Chemical 23(2-3): 135-156.

2. Faia PM, Jesus EL, Louro CS (2014) $\mathrm{TiO}_{2}: \mathrm{WO}_{3}$ composite humidity sensors doped with $\mathrm{ZnO}$ and $\mathrm{CuO}$ investigated by impedance spectroscopy. Sensors Actuators B: Chemical 203: 340-348.

3. Faia PM, Libardi J, Louro CS (2016) Effect of $\mathrm{V}_{2} \mathrm{O}_{5}$ doping on p-to-n conduction type transition of $\mathrm{TiO}_{2}: \mathrm{WO}_{3}$ composite humidity sensors. Sensors and Actuators B: Chemical 222: 952-964.

4. Araújo ES, Libardi J, Faia PM, Oliveira HP (2017) Humidity-sensing properties of hierarchical $\mathrm{TiO}_{2}: \mathrm{ZnO}$ composite grown on electrospun fibers. Journal of Materials Science: Materials in Electronics 28(21): 16575-16583.

5. Araújo ES, Libardi J, Faia PM, Oliveira HP (2018) Characterization and electrical response to humidity of sintered polymeric electrospun fibers of vanadium oxide- $\left(\mathrm{TiO}_{2} / \mathrm{WO}_{3}\right)$. Journal of Electronic Materials $47(5)$ : 2710-2717. 
Creative Commons Attribution 4.0 International License

For possible submissions Click Here

\section{RDMS}

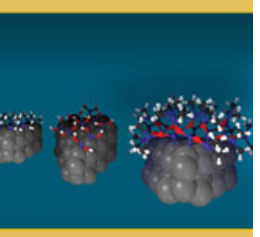

\section{Research \& Development in Material Science}

\section{Benefits of Publishing with us}

- High-level peer review and editorial services

- Freely accessible online immediately upon publication

- Authors retain the copyright to their work

- Licensing it under a Creative Commons license

- Visibility through different online platforms 